\title{
Vorwort zur deutschsprachigen Ausgabe
}

Ein Buch sollte sich eigentlich von selber einführen, aber gewisse Erläuterungen hinsichtlich der Absichten des Autors könnten für den Leser von Vorteil sein. Beim Niederschreiben der tschechischen Ausgabe war ich bestrebt, die Grundbegriffe der allgemeinen und physikalischen Chemie, auf die ein Arzt oder Biologe beim Studium der Literatur stoßen könnte, in einer möglichst zugänglichen Form zu erklären. Das Buch hatte auch unter Pharmazeuten, den Biochemikern selbst und unter Studenten Anklang gefunden. Ich entschloß mich deshalb den Text für die neue Ausgabe, unter Beibehaltung der ursprünglichen Gliederung des Stoffes, zu erweitern und so umzuarbeiten, damit er für andere Biowissenschaftler und für Lehrzwecke von Nutzen sein könnte. Der Umfang des Buches, vor dem der Leser zurückschrecken kann, ist nicht etwa durch Anhäufung eines Übermaßes an Informationen bedingt; er war im Gegenteil aus zwei Gründen notwendig. Erstens ist es nicht möglich einen maximal kurz gefaßten Text so niederzuschreiben, daß er mannigfaltigen, wenn auch in vieler Hinsicht sich sehr nahe stehenden Fachgebieten, zweckdienlich sein und Leser mit verschiedenen Ansprüchen, wie Forscher und Studenten, befriedigen könnte. Die einzig mögliche Lösung führte zu einem umfangreicheren Buch in dem sich ein jeder die Abschnitte auswählen kann, die für ihn von Interesse sind und das ihm zusagende Ausmaß besitzen.

Ich versuchte diesen Anforderungen durch die Gliederung des Buches, die Anwendung des Petits für auslegende und ergänzende Partien, häufige, im Text angeführte Hinweise auf eingehendere Erklärungen bringende Kapitel und durch ein eingehendes Register nachzukommen. Der zweite Grund dafür, daß der Inhalt des Buches nicht in knapper Form niedergeschrieben wurde, wo nur das Notwendigste vorhanden wäre, was der Leser aus der allgemeinen und physikalischen Chemie wissen sollte, war das Bestreben einen verständlichen Text zu schaffen. Zwar ist es gerade in der physikalischen Chemie möglich, sich mit Hilfe zutreffender Definitionen und mathematischer Beziehungen sparsam auszudrücken, doch ist diese Art für den in der Mathematik und Physik wenig Bewanderten schwer verständlich. Deshalb habe ich oft bildhafte Darstellungen mit vielen Worten gewählt, wo sonst eine knappe Definition oder eine einfache mathematische Gleichung vollauf genügt hätte.

Außer einer Reihe von Kollegen, die mir bei der Vorbereitung der tschechischen Ausgabe behilflich waren, bin ich für die Hilfe bei der Umarbeitung des Textes, seiner Übersetzung, fachlichen und sprachlichen Revision besonders Herrn Prof. RNDr. J. KORYTA, DrSc., Herrn Ing. P. MITSCHKA, CSc., Frau Dr. H. DURÉ-BAŽANTOVÁ von der Tschechosl. Akademie der Wissenschaften, weiter Herrn Dr.-Ing. $H$. W. SICHTING von der Technischen Universität zu Berlin und Herrn RNDr. W. WANEK, CSc. von der pädagogischen Fakultät in Ústí $n / L$. zu Dank verpflichtet. Nicht zuletzt 
gehört mein Dank auch Frau RNDr. J. PR̆IBYLOVA, CSc., Herrn RNDr. I. KALOUSEK und Frau RNDr. E. ŘEǨ́BKOVÁ, CSc. vom Institut für Hämatologie und Bluttransfusion in Prag. Herrn Prof. M. F. PERUTZ aus dem Medical Research Council Laboratory of Molecular Biology Cambridge (England) möchte ich für die liebenswürdige Überlassung der Photographie des Modells des Hämoglobinmoleküls (Bild 1.41) meinen Dank aussprechen. Dem Verlag Avicenum und dem Verlag Walter de Gruyter danke ich für verständnisvolle Zusammenarbeit.

Prag 1976

ZDENĚK VODRÁŽKA 